\title{
Multicentre variability of MRI-based medial temporal lobe volumetry in
}

\section{Alzheimer's disease}

Stefan J. Teipel ${ }^{1,2}$, Michael Ewers ${ }^{1,3}$, Stefanie Wolf ${ }^{4}$, Frank Jessen ${ }^{5}$, Heike Kölsch ${ }^{5}$,

Sönke Arlt ${ }^{6}$, Christian Luckhaus ${ }^{7}$, Peter Schönknecht ${ }^{8,9}$, Klaus Schmidtke $^{10}$, Isabella Heuser ${ }^{11}$, Lutz Frölich $^{12}$, Gabriele Ende ${ }^{12}$, Johannes Pantel ${ }^{13}$, Jens Wiltfang ${ }^{15}$, Christine Born ${ }^{16}$, Johannes Kornhuber ${ }^{15}$, Harald Hampel ${ }^{1,3}$

${ }^{1}$ Department of Psychiatry, Ludwig-Maximilian University Munich, Germany

${ }^{2}$ Department of Psychiatry, University Rostock, Germany

${ }^{3}$ Discipline of Psychiatry, School of Medicine, Trinity College Dublin, Ireland

${ }^{4}$ Department of Psychiatry, University Göttingen, Germany

${ }^{5}$ Department of Psychiatry, University Bonn, Germany

${ }^{6}$ Department of Psychiatry, University of Hamburg, Germany

${ }^{7}$ Department of Psychiatry, University of Düsseldorf, Germany

${ }^{8}$ Department of Psychiatry, University of Heidelberg, Germany

9Department of Psychiatry, University Leipzig, Germany

${ }^{10}$ Department of Psychiatry, University of Freiburg, Germany

${ }^{11}$ Department of Psychiatry, Free University Berlin, Germany

${ }^{12}$ Central Institute of Mental Health, Mannheim, Germany

${ }^{13}$ Department of Psychiatry, University of Frankfurt, Germany

${ }^{14}$ Department of Psychiatry, University Essen

${ }^{15}$ Department of Clinical Radiology, Ludwig-Maximilian University, Munich, Germany

${ }^{16}$ Department of Psychiatry, University of Erlangen, Erlangen, Germany

Running Title: Multicentre hippocampus volumetry in AD

\section{Manuscript requirements:}

Title: $\quad 90$ spaces

Abstract: $\quad 219$

Text: $\quad 4460$

References: $\quad 52$

Figures: 4

Tables: $\quad 5$

\section{Corresponding Author:}

Stefan J. Teipel, M.D.

Alzheimer Memorial Centre and Geriatric Psychiatry Branch

Dementia and Neuroimaging Section,

Department of Psychiatry,

Ludwig-Maximilian University,

Nussbaumstr.7,

80336 Munich, Germany

Tel.: +49-89-5160-5860

Fax: $+49-89-5160-5865$ 
E-mail: stefan.teipel@med.uni-muenchen.de 


\begin{abstract}
MRI based volumetry of the hippocampus and amygdala has been suggested as a biomarker of Alzheimer's disease (AD). For validation of the candidate marker, however, effects of multicentre acquisition on measurement variability have to be considered. In the present study we determined effects of multicentre acqusitions of MRI data across 12 clinical sites within the German Competence Network on Dementias in 113 patients with clinically probable AD and 150 patients with amnestic mild cognitive impairment (MCI). Hippocampus and amygdala volumes were significantly reduced in $\mathrm{AD}$ compared to $\mathrm{MCI}$ patients using data pooled across centres. The between-centre effect accounted for 5 to $15 \%$ of within-centre variability. Using mixed effects regression, we found specific correlations between delayed recall of verbal and non-verbal material and global cognitive measures with hippocampus and amygdala volumes. In contrast drawing performance was not correlated with volume measures. ApoE4 genotype had no effect on volumetric measures. Power analysis for the detection of a difference in the volumes between $\mathrm{AD}$ and MCI patients across centres showed that multicentre variability did not significantly affect effect sizes. The total sample size needed is $\mathrm{N}=58$ for hippocampus and $\mathrm{N}=158$ for amygdala volumes. Our data indicate that multicentre acquisition of MRI data using manual volumetry is reliable and feasible for diagnostic trials and replicates essential findings from smaller scale monocentre studies.
\end{abstract}




\section{Introduction}

MRI-based volumetry of medial temporal lobe regions, especially of the hippocampus, is among the best established biomarker candidates of Alzheimer's disease (AD) to date. The majority of experimental studies so far employed manual volumetric techniques to determine volumes of hippocampus, amygdala, entorhinal cortex, and other subregions of medial temporal lobe[12,25,50]. Few studies have employed semi-automated techniques to determine hippocampus volume, accounting for more than $80 \%$ of variation in manual measurements[7,21]. Manual hippocampus volume reaches about $70 \%$ accuracy $[12,26]$ in the prediction of $\mathrm{AD}$ in patients with mild cognitive impairment (MCI), an at risk group of $\mathrm{AD}[32]$, and between 80 to $90 \%$ accuracy in the discrimination between patients with $\mathrm{AD}$ and healthy control subjects[17,18]. Levels of accuracy are similar for amygdala volumetry, but less well established in comparison to hippocampus volumetry[50].

In several phase II clinical trials specificity of clinical criteria for MCI was not sufficient to select a high risk population to show secondary preventive treatment effects with high power. Therefore, hippocampus and amygdala volumetry has been proposed as non-invasive and widely available marker to select and stratify at risk subjects for clinical trials[8]. The expectation is that the effort of manual or semi-automated volumetry would be paid off by the risk enrichment of the study sample leading to high testing power. It remains to be shown, however, if acquisition of MRI data derived from multiple scanners increases variability of volumetric measures and how this would affect power calculations for a diagnostic trial.

Besides their increasing importance in clinical trials, multicentre acquisitions give access to relatively large sample sizes for answering questions on the association between specific cognitive impairment and regional atrophy. However, studies using samples derived from multiple sites have not considered centre effects[28]. The same is true for studies on the effect of ApoE4 genotpye, the best established genetic risk factor for sporadic $\mathrm{AD}[10]$, on hippocampus and amygdala volumes[15]. Therefore, it is not yet clear whether multicentre 
acquisition allows replicating effects of impairment in specific cognitive domains on hippocampus and amgydala volumes and may help to resolve the still controversial discussion on the effect of ApoE4 genotype on medial temporal lobe volumes in AD and MCI. To answer these questions, statistical models need to employ random effect terms to control for the hierarchical covariance structure of multicentre data, with higher covariance within than between centres [6]. However, these models have only rarely been employed in MRI multicentre designs so far.

Here we present data derived from a large-scale prospective multicentre trial within the German Dementia Network where we determined volumes of the hippocampus and the amygdala in a sample of $\mathrm{AD}$ and $\mathrm{MCI}$ patients acquired at 12 University-based clinical centres across Germany. We report multicentre variability and its effect on effect size estimates using bootstrapping to determine the confidence interval for a multicentre effect size. Additionally, we determine effects of cognitive impairment and ApoE genotype on hippocampus and amygdala volume using random effects models to explicitly model variation in effects across centres. 


\section{Methods}

\section{Subjects}

Out of 400 patients with MCI or dementia due to a large range of conditions, including cerebrovascular disease, neurodegenerative disorders such as AD, LBD and FTD, and secondary dementias, we selected those 113 patients that had the clinical diagnosis of probable AD according to NINCDS-ADRDA criteria, and those 150 patients that had the clinical diagnosis of amnestic mild cognitive impairment according to Mayo Clinic criteria[41]. These 400 subjects were the first in a series of 975 patients that had prospectively been included in the first wave of the diagnostic study of the German Dementia Network between August 2003 and December 2005. This first series was selected because scans of these subjects had been measured by 4 raters (100 scans per rater) where diagnoses and centres were balanced across raters. The mean age was significantly different, but gender distribution and years of education were not significantly different between groups (table 1). Severity of cognitive impairment has been assessed using the Mini-Mental state examination score[16]. As expected, groups differed significantly in MMSE-scores (table 1).

ApoE genotype was available in a subgroup of $88 \mathrm{AD}$ patients and $122 \mathrm{MCI}$ patients. Age, gender distribution, MMSE scores and years of education were not different between the subgroup with ApoE genotype and the subjects without this measure (table 1).

- Table 1 near here -

Patients were recruited from 12 centres across Germany, the number of subjects per centre and diagnostic group is listed in table 4. The distribution of sample size and diagnoses across centres was not homogeneous, with $\chi^{2}=24.5,11 \mathrm{df}, \mathrm{p}=0.01$. The study was approved by the Central IRB Board of the Network located at the University of Mannheim and by each of the local IRBs of the participating centres.

\section{Psychometric testing}


Patients underwent psychometric testing including the MMSE score[16], immediate and delayed recall of the Wechsler Memory-Revised Version logical memory[51], word list learning and free recall of word lists as well as drawing of increasingly complex figures and free recall of drawings from the Consortium to Establish a Registry for Alzheimer's Disease (CERAD) cognitive battery[39], the Alzheimer Disease Assessment Scale (ADAS) [46]and the clinical dementia rating (CDR)[38]. Due to low variability in global CDR scores (0.5 in MCI and 0.5 to 1.0 in $\mathrm{AD}$ patients), we only used the CDR sum of boxes for correlation analyses. All tests were applied by trained psychometricians after initial central rater training that was repeated annually.

\section{MRI acquisitions}

MRI scans were obtained on a 1.5 Tesla scanner in all centres, where the hardware and sequence parameters varied between centres. Siemens Scanners were used (Siemens Sonata or Siemens Magnetom Vision) at 8 centres and Philips Scanners (Philips Gyrosscan and Philips Intera) at the remaining 4 centres. MRI scans were conducted with a sagittal magnetization prepared rapid gradient echo (MPRAGE sequence) on Siemens Scanners and a 3D fast T1weighted gradient echo sequence on Philips Scanners. Among all centres the TR varied between 9.3 and $20 \mathrm{~ms}$ and TE between 3.93 and $4.38 \mathrm{~ms}$. These sequences served as basis for the volumetric analysis. Additionally, each subject underwent an axially oriented FLAIR-sequence for exclusion of subcortical lesions.

\section{Volumetry}

Hippocampus and amygdala volumes were determined according to a previously published protocol[45,50]. Prior to volumetric measurements, all MR volumes were corrected for image intensity non-uniformities, mapped by affine stereotaxic transformation into coordinates based on the Talairach Atlas, resampled onto a $1 \mathrm{~mm}$ voxel-grid, and each second scan was flipped in the left-right orientation. These preprocessing steps reduce inter-scanner variability due to scan artifacts, correct for effects of whole brain atrophy and preclude rater- 
bias for the left or the right hemisphere. We calculated the determinant of the 12-parameter affine transformation matrix which represents the overall volume effect associated with the transformation of the brain into standard space. After hippocampus volumetry in standard space we divided the regional volumes by the determinant of the individual affine transformation matrix to determine volumes in native space. Native space volumes were the basis for subsequence statistical analysis.

Volumetric analyses were performed with the interactive software package Display, developed at McConnell Brain Imaging Centre at the Montreal Neurologic Institute, by 4 raters blinded to subjects identity, clinical diagnosis and left / right orientation of scans. The number of scans per rater was balanced for centre and diagnosis. The anatomical boundaries used for segmentation of hippocampus and amygdala have been described in detail in a previous study[45]. The intra-class correlation coefficients were 0.92 for right and 0.93 for left hippocampus, and 0.69 for right and 0.80 for left amygdala, when the 4 raters measured medial temporal lobe volumes independently in 10 randomly selected MRI scans.

\section{Statistics}

As the data were clustered within centres and raters, we employed a linear mixed effects regression model with the volumetric measures as dependent variables, diagnosis, age and gender as independent fixed effects and rater and centre as well as the the two-way interactions of rater and centre with diagnosis, gender and age independent random effects. This model takes into account that the observations from the same centre and the same rater are correlated, i.e. not independent as assumed in a simple fixed effect linear model (such as ANOVA). Moreover, modelling centre and rater as random effects allows drawing inference about effects of diagnosis that apply to an entire population of centres and raters, not only to the particular centres and raters in the data set. Following the same line of argumentation, we used mixed effects regression models to determine effects of ApoE4 genotype (coded as $1=$ 
at least one Apo E4 and $0=$ no Apo E4) and cognitive measures on volumes including centre and rater and the respective interaction effects as random effects, controlling for age, gender, diagnosis and the interaction of diagnosis with cognitive scores and ApoE4 genotype as fixed effects. Models were calculated using Procedure MIXED in SAS 9.1[31].

The effect size d [9] of the difference in the volumes of hippocampus and amygdala between $\mathrm{AD}$ and $\mathrm{MCI}$ patients was estimated according to the following formula: $d=\frac{(x-y)}{\delta_{x y}}$

where $\mathrm{x} / \mathrm{y}$ is the difference in the mean volume of hippocampus and amygdala between $\mathrm{AD}$ and MCI patients, and $\delta_{\mathrm{xy}}$ the pooled standard deviation of volumes of hippocampus and amygdala, respectively, obtained from $\mathrm{AD}$ and MCI patients.

Due to the small sample sizes in some centres, we used the Mann-Whitney U test for comparisons of volumes between groups within centres. The corresponding effect sizes were determined for each centre separately (henceforth, referred to as "monocentre effect size"). In addition, the effect size of the group difference on the basis of hippocampus and amygdala volumes pooled across centres was estimated (henceforth, referred to as "pooled effect size"). In order to compare the monocentre effect size to a multicentre effect size, $n=10$ data points were randomly sampled each from the MCI group $(n=150)$ and AD group $(n=113)$ pooled among centres and the effect size of the group difference was calculated. The random sampling of hippocampus and amygdala volumes from each group and the calculation of the respective effect size was iterated 10,000 times. The mean effect size of the resulting sampling distribution along with the $95 \%$ confidence interval was calculated using a customized program written in MATLAB 5.6. We chose to sample $\mathrm{n}=10$ in each sampling trial applied to the pooled data since this sample size approximates the one collected at each single centre, thus allowing for a comparison between the monocentre and multicentre effect size. A one sample t-test was used to test whether the monocenter effect sizes deviated 
significantly from the mean value of the multicentre effect size. The sample size required to detect a pooled effect size with a statistical power of 0.9 , at $\mathrm{p}=0.05$ for a two-tailed t-test, was estimated using the computer program G*POWER 3 (available online at http://www.psycho.uni-duesseldorf.de/abteilungen/aap/gpower3/)[13]. We determined differences in measurement variability between amygdala and hippocampus using the coefficient of variation as estimate of within rater variation and the ratio of between to within rater variability as estimate of between rater variation. The coefficient of variation was computed by dividing the standard deviation of the measurements by the mean value of the measurements averaged across raters. 


\section{Results}

In the linear mixed models, there were significant effects of diagnosis on bilateral hippocampus and left amygdala volumes (smaller volumes in AD patients), for age on bilateral hippocampus (smaller volumes in older subjects), and for gender on right hippocampus and bilateral amygdala volumes (smaller volumes in women) (table 2). When we controlled the models for the determinants of the transformation matrices of each scan from native to standard space, representing the effect of overall brain volume, effects of age and diagnosis remained unchanged, however, effects of gender were no more significant, suggesting that the effect of gender is accounted for by overall brain volume.

- Table 2 near here -

Subsequently, we determined the effect of cognitive performance and ApoE genotype on volumes. All comparisons were controlled for age, gender, diagnosis and the interaction effect of diagnosis by cognitive scores and ApoE genotype, respectively, as fixed effects and rater and centre and the interaction of rater and centre with cognitive scores and ApoE genotype, respectively, as random effects. There was a significant effect of free recall of verbal material, and immediate and delayed logical memory on all volumes (table 3). Recall of previously drawn figures was significantly correlated with bilateral hippocampus and right amygdala volume, whereas drawing itself was not correlated with any volumetric measure. Among global cognitive measures, MMSE score was significantly correlated with all volumetric measures, ADAScog sum score was associated with left hippocampus and bilateral amygdala volumes, and CDR sum of boxes score was related to bilateral hippocampus and right amygdala volumes (table 3). There was no significant effect of Apo E4 carrier status on any volumetric measure (table 3). When we controlled the models for the determinants of the transformation matrices of each scan from native to standard space, representing the effect of overall brain volumes, significance of effects remained unchanged.

- Table 3 near here - 
Mean volumes of hippocampus and amygdala in $\mathrm{AD}$ and $\mathrm{MCI}$ patients and the monocentre effect sizes of group differences for each centre are displayed in table 4. At $p<$ 0.05 , volumes of left hippocampus were significantly different between groups within 7 of 12 centres, of right hippocampus within 6 of 12 centres, of left amygdala within 2 of 12 centres and of right amygdala within 1 of 12 centres (table 4).

- Table 4 near here -

The mean of the sampling distribution of the multicentre effect sizes and the pooled effect sizes were nearly identical for all volumes (table 5 and figures 1 to 4 ). The total sample sizes required to show a significant difference between groups at $\mathrm{p}<0.05$ with $90 \%$ power ranged between 52 patients ( $=26$ per group) for right hippocampus and 158 (=79 per group) for right amygdala (table 5).

- Table 5 and Figures 1 to 4 near here -

For left hippocampus two monocentre effect sizes (centres I and XII) and for left amygdala three monocentre effect sizes (centres V, VII, and XII) were outside the 95\% confidence interval of the multicentre sampling distribution (table 4 and figures 1 to 4 ) Among the outlying centres, only centre VII had been outside the American College of Radiology (ACR) criteria for phantom measurements upon first evaluation, but met all criteria at follow-up[14]. All outlying centres, however, had sample sizes below 6 subjects in at least one diagnostic group. A one-sample t-test confirmed that the monocentre effect sizes did not differ in either direction from the mean multicentre effect size (11 degrees of freedom for all tests: left hippocampus, $\mathrm{p}=0.38$; left amygdala, $\mathrm{p}=0.72$; right hippocampus, $\mathrm{p}=0.36$; right amygdala, $\mathrm{p}=0.47$ ), suggesting that the monocentre effect sizes were not superior when compared to the multicentre effect sizes.

The coefficients of variation ranged between 0.21 and 0.23 for amygdala and between 0.20 and 0.23 for hippocampus. The proportion of between rater variability to within rater 
variability ranged between $15 \%$ and $38 \%$ for amygdala and between $24 \%$ and $27 \%$ for hippocampus. 


\section{Discussion}

The aim of the current study was to investigate the variability of medial temporal lobe volume measurements across multiple sites and the effect of this variability on estimates of required sample sizes to demonstrate significant group differences. Additionally, we studied effects of cognitive performance and Apo E4 genotype on medial temporal lobe volumes using a mixed effects approach to explicitly model centre effects.

We found significant reductions of the volumes of hippocampus and amygdala in AD compared to MCI patients. This finding agrees with previous studies reporting significant reductions of hippocampus volumes in AD compared to MCI patients [40] and with longitudinal studies [22] suggesting that hippocampus volume continues to shrink even in manifest stages of $\mathrm{AD}$. One previous study reported reduced amygdala volumes in $\mathrm{AD}$ compared to MCI patients[5]. The effect size for amygdala volume reduction was -1.0 for left and -1.1 for right amygdala in this previous study which is within the expected range of effect size estimates as defined by the $95 \%$ confidence limits of the sampling distribution of the multicentre effect sizes from our study (figures 2 and 4). To our knowledge, no longitudinal studies have been reported on rates of atrophy of amygdala in $\mathrm{AD}$ over time. Based, however, on this earlier study and our data, one would expect that similar to hippocampus atrophy the amygdala continue to shrink in manifest stages of $\mathrm{AD}$. In contrast to the majority of earlier studies, we used MRI data collected across several centres. Therefore, we employed a mixed effects linear model to take into account that data were clustered within centres with higher covariance within than between centres. Different to fixed effects models, mixed effects models allow drawing inference not only on the particular set of centres and raters involved, but on a whole population of centres and raters[49]. Earlier studies have used multicentre data as well, but either did not consider centre effects at all [28] or used a fixed effects model that does not allow for variation of effects between centres[24]. 
Taking centre and rater effects into account, we found significant effects of age on hippocampus, but not amygdala volumes. Correlations between hippocampus volumes and age have been described in a large range of previous studies. It has been proposed that the inverse correlation between age and hippocampus volumes reflects an effect of disease duration. Greater age would on average correspond to greater disease duration, which in turn will correspond to more atrophic hippocampi[24]. However, the lack of an effect for amygdala makes this interpretation more unlikely, since amygdala atrophy also is expected to increase with disease duration[11]. The clear discrepancy of age effects between hippocampus and amygdala therefore deserves further study on potential mechanisms other than disease duration. Similar to earlier studies, we found smaller regional volumes of hippocampi and amygdala volumes in women than in men[1,25,44]. However, this effect was determined by overall brain size (represented by the determinant of the affine transformation matrix in standard space) and did not represent a specific gender effect on regional medial temporal lobe volumes in $\mathrm{MCI}$ and $\mathrm{AD}$.

We have not found an effect of ApoE4 genotype on hippocampus and amygdala volumes in AD or MCI. Findings from earlier studies are ambiguous. Some studies, did not find an effect of ApoE4 genotype[23], other studies reported significant effects of ApoE4 genotype on hippocampus volume in $\mathrm{AD}[19,30]$. To explain this discrepany, it has been argued that subject numbers had been too low in the negative studies. This argument does not hold, however, given the subject numbers in this and one previous study[23]. Cohens $d$ in one relatively large positive study comprising 138 patients was $0.55[19]$. Our study was powered to detect an effect size of 0.55 at $80 \%$ power and a $\mathrm{p}$ value of 0.05 in the $\mathrm{AD}$ group. The ApoE effects in our sample lay far below this effect size. One may assume that sample characteristics, such as age, gender or cognitive status may contribute to discrepancies between studies. However, age, gender and MMSE scores were matched between the AD patients with and without ApoE4 genotype both in our sample and in the previous study 
showing a significant effect of ApoE4[19]. Similar to us, one earlier study found no effect of ApoE4 genotype on amygdala volume [30] in AD patients. However, two studies found significantly larger atrophy of amygdala in Apo E4 carriers compared to non-carriers[3,19].

Controversial findings have been reported on the effect of ApoE4 on hippocampus volumes in MCI, as well. One large multicentre trial included a subgroup of $267 \mathrm{MCI}$ patients with MRI and ApoE4 genotyping. These subjects had been recruited from 65 sites in 14 countries. The authors found highly significant effects of ApoE4 genotype on hippocampus volumes. However, the authors did not consider centre effects at all[15], rendering the results difficult to interpret. The study does not provide data to calculate effect sizes. In conclusion, to date the question of the effect of ApoE4 genotype on hippocampus and amygdala atrophy in $\mathrm{AD}$ and $\mathrm{MCI}$ remains still unresolved. However, our data support the notion that other factors than sample size and sample characteristic, such as age, gender and general cognitive status, contribute to the discrepancies between studies.

When we accounted for centre and rater effects, we found significant correlations between free recall of verbal material and of drawings as well as immediate and delayed recall of logical memory with bilateral hippocampus and amygdala volumes. In contrast, verbal learning was not related to hippocampus volume, drawing performance was not related to hippocampus and amygdala volumes. Among global cognitive measures, MMSE score was correlated with all volumetric measures, ADAScog and CDR sum of boxes mainly with hippocampus volumes. These results were independent of the determinant of the affine transformation matrix as a global measure of brain size.

Our findings suggest a specific correlation between free recall of verbal and non verbal material with hippocampus and amygdala volumes. They agree with several earlier studies showing correlations between memory related cognitive measures and hippocampus [42] and amygdala $[4,35,37]$ volume. In agreement with earlier studies[27,28], we found significant correlations of hippocampus volumes with delayed free recall, but not with immediate 
memory of a word list, supporting the specific role of hippocampus in the consolidation rather than encoding of new material[48]. Interestingly, however, not only delayed, but also immediate logical memory was correlated with hippocampal and amygdala volumes. This agrees with a study in patients with intractable temporal lobe epilepsy. Those patients having a focus in the left medial temporal lobe showed both impaired immediate and delayed logical memory[36]. However, when we assessed a linear model including both immediate and delayed logical memory, only delayed, but not immediate memory was significantly correlated with hippocampus volumes. This finding suggests that the effects for logical memory are dominated by the delayed recall. The significant correlation between recall of drawings independently of the immediate drawing performance agrees with the prominent role of the hippocampus in the consolidation of a memory.

The differences between global cognitive measures (MMSE, ADAScog, CDR-SB) in their correlations with volumes may be related to the amount of variance of each scale that is explained by memory. We applied a principal component analysis to the cognitive measures in our MCI and AD samples. Within both groups, memory tests, MMSE and ADAScog, but not the CDR-SB showed a high positive loading on the first component accounting for about $39 \%$ of variance in the MCI and $26 \%$ of variance in the AD group.

In summary, our data replicate earlier findings on specific correlations between memory performance and medial temporal atrophy using a multicentre approach with mixed effects regression.

Based on the observed multicentre variability of volumetric measures, one might have expected a reduced effect size for multicentre data when compared to monocentre data. However, in our data the multicentre effect size did not systematically differ from the monocentre effect sizes. The pooled effect size and the average of the sampling distribution of the multicentre effect size were nearly identical. This suggests that the effect of between centre variability on effect sizes between groups is rather limited. One earlier study 
investigated variability of hippocampus volumetry across multiple centres. The authors studied variability of percentile position of hippocampus volume from $\mathrm{AD}$ patients on a normal distribution of healthy elderly subjects across 38 centres participating in a clinical trial[24]. They found a significant effect of centre on percentile position, with variability arising form differences between centres constituting about $5 \%$ of variability arising from differences between subjects within centres. In our data, the proportion of total variability arising from centre was about $7.5 \%$ for hippocampus and about $4.5 \%$ for amygdala in AD and about $15 \%$ for hippocampus and about $7 \%$ for amygdala in MCI. Thus the centre-induced variability in our $\mathrm{AD}$ group is quite similar to the variability observed in the previous study[24]. We can not finally explain why centre effects were higher in the MCI than in the $\mathrm{AD}$ patients. One factor, however, may be that clinical criteria of MCI are less stringent than those of clinical AD leaving more room for biased selection of patients at each single site.

Pooled and averaged multicentre effect sizes were nearly identical. This again supports a limited effect of between centres variability on overall effect sizes. Sample sizes required to show a significant between group difference (at $\mathrm{p}<0.05$ and $90 \%$ power) were about 58 subjects (29 per group) for hippocampus and 158 subjects (79 per group) for amygdala volumes. The difference between hippocampus and amygdala in required sample sizes may partially result from true biological differences in the extent of atrophy between both regions. There is some, but still very limited, evidence that neuron loss may be more pronounced and neurofibrillary tangles may be more frequent in amygdala than in hippocampus in early stages of $\mathrm{AD}[2,20,33,34,43,47,52]$. This may indicate more rapid progression or earlier onset of pathological changes in amygdala than hippocampus so that amygdala atrophy may have reached its maximum even before the conversion from MCI to AD.

Another reason for differences in effect sizes between markers may be differences in measurement variability. However, variation of measurements within raters and the 
contribution of between rater variability to within rater variability were homogeneously distributed between amygdala and hippocampus measurements.

For the interpretation of the comparison of monocentre and multicentre effect sizes some caveats need to be considered as well. One has to note that the estimation of the monocentre effect sizes was based on a rather small sample size per centre. Although the effect of diagnosis based upon the pooled multicentre data was statistically significant, this was true for not all of the within-centre effect sizes of group differences. Indeed effect sizes that lay outside the $95 \%$ confidence interval of the sampling distribution of the multicentre effect size were associated with those centres that had the smallest sample size. Thus, it is possible that the rather large variability within centres due to low sample size may have occluded variability between centres. The multicentre effect may have become detectable if larger sample sizes per centre had been included. Using mixed effects regression with restricted maximum likelihood estimation [29] as in the present study weights centre effects by sample size such that observations with fewer data points contribute less to the overall estimate of centre effects than centres with larger sample size.

The main focus of research on diagnostic markers is on the separation of AD patients from healthy subjects and on the discrimination between MCI patients that convert to AD and MCI patients that remain stable. For a study aiming to differentiate between MCI and AD patients at the interface between functionally independent and dependent living in the community, it will be important to determine whether multicentre MRI captures essential features of between group differences in brain morphology. Our data indicate that multicentre acquisition of MRI does not strongly affect volumetric measures and their effect sizes, supporting the approach of ongoing national and international networks to collect multicentre MRI data to establish MRI as a reliable biomarker of AD. 


\section{Acknowledgement}

The study was funded by a grant from the Bundesministeriums für Bildung und Forschung (BMBF 01 GI 0102) awarded to the dementia network "Kompetenznetz Demenzen". 


\section{References}

[1] Apostolova LG, Dinov ID, Dutton RA, Hayashi KM, Toga AW, Cummings JL, Thompson PM. 3D comparison of hippocampal atrophy in amnestic mild cognitive impairment and Alzheimer's disease. Brain 2006;129(Pt 11):2867-73.

[2] Ball MJ. Neuronal loss, neurofibrillary tangles and granulovacuolar degeneration in the hippocampus with ageing and dementia. A quantitative study. Acta Neuropathol (Berl) 1977;37(2):111-8.

[3] Basso M, Gelernter J, Yang J, MacAvoy MG, Varma P, Bronen RA, van Dyck CH. Apolipoprotein E epsilon4 is associated with atrophy of the amygdala in Alzheimer's disease. Neurobiol Aging 2006;27(10):1416-24.

[4] Basso M, Yang J, Warren L, MacAvoy MG, Varma P, Bronen RA, van Dyck CH. Volumetry of amygdala and hippocampus and memory performance in Alzheimer's disease. Psychiatry Res 2006;146(3):251-61.

[5] Bottino CM, Castro CC, Gomes RL, Buchpiguel CA, Marchetti RL, Neto MR. Volumetric MRI measurements can differentiate Alzheimer's disease, mild cognitive impairment, and normal aging. Int Psychogeriatr 2002;14(1):59-72.

[6] Brown H, Prescott R. Applied mixed models in medicine. Chichester, UK: John Wiley \& Sons Ltd; 1999.

[7] Carmichael OT, Aizenstein HA, Davis SW, Becker JT, Thompson PM, Meltzer CC, Liu Y. Atlas-based hippocampus segmentation in Alzheimer's disease and mild cognitive impairment. Neuroimage 2005;27(4):979-90.

[8] Chetelat G, Baron JC. Early diagnosis of Alzheimer's disease: contribution of structural neuroimaging. Neuroimage 2003;18(2):525-41.

[9] Cohen J. Statistical Power Analysis for the Behavioural Sciences. New York: Academic Press; 1977. 
[10] Corder EH, Saunders AM, Strittmatter WJ, Schmechel DE, Gaskell PC, Small GW, Roses AD, Haines JL, Pericak-Vance MA. Gene dose of apolipoprotein E type 4 allele and the risk of Alzheimer's disease in late onset families. Science 1993;261(5123):921-3.

[11] Cuénod C-A, Denys A, Michot J-L, Jehenson P, Forette F, Kaplan D, Syrota A, Boller F. Amygdala atrophy in Alzheimer's disease. An in vivo magnetic resonance imaging study. Arch Neurol 1993;50:941-5.

[12] Devanand DP, Pradhaban G, Liu X, Khandji A, De Santi S, Segal S, Rusinek H, Pelton GH, Honig LS, Mayeux R, Stern Y, Tabert MH, de Leon MJ. Hippocampal and entorhinal atrophy in mild cognitive impairment: prediction of Alzheimer disease. Neurology 2007;68(11):828-36.

[13] Erdfelder E, Faul F, Buchner A. GPOWER: A general power analysis program. Behavior Research Methods, Instruments \& Computers 1996;28:1-11.

[14] Ewers M, Teipel SJ, Dietrich O, Schonberg SO, Jessen F, Heun R, Scheltens P, van de Pol L, Freymann NR, Moeller HJ, Hampel H. Multicenter assessment of reliability of cranial MRI. Neurobiol Aging 2006;27(8):1051-9.

[15] Farlow MR, He Y, Tekin S, Xu J, Lane R, Charles HC. Impact of APOE in mild cognitive impairment. Neurology 2004;63(10):1898-901.

[16] Folstein MF, Folstein SE, McHugh PR. Mini-mental-state: a practical method for grading the cognitive state of patients for the clinician. J Psychiatr Res 1975;12:18998.

[17] Golebiowski M, Barcikowska M, Pfeffer A. Magnetic resonance imaging-based hippocampal volumetry in patients with dementia of the Alzheimer type. Dement Geriatr Cogn Disord 1999;10(4):284-8. 
[18] Hampel H, Teipel SJ, Bayer W, Alexander GE, Schwarz R, Schapiro MB, Rapoport SI, Moller HJ. Age transformation of combined hippocampus and amygdala volume improves diagnostic accuracy in Alzheimer's disease. J Neurol Sci 2002;194(1):15-9.

[19] Hashimoto M, Yasuda M, Tanimukai S, Matsui M, Hirono N, Kazui H, Mori E. Apolipoprotein E epsilon 4 and the pattern of regional brain atrophy in Alzheimer's disease. Neurology 2001;57(8):1461-6.

[20] Herzog AG, Kemper TL. Amygdaloid changes in aging and dementia. Arch Neurology 1980;37(10):625-9.

[21] Hsu YY, Schuff N, Du AT, Mark K, Zhu X, Hardin D, Weiner MW. Comparison of automated and manual MRI volumetry of hippocampus in normal aging and dementia. J Magn Reson Imaging 2002;16(3):305-10.

[22] Jack CR, Jr., Petersen RC, Xu Y, O'Brien PC, Smith GE, Ivnik RJ, Boeve BF, Tangalos EG, Kokmen E. Rates of hippocampal atrophy correlate with change in clinical status in aging and AD. Neurology 2000;55(4):484-9.

[23] Jack CR, Jr., Petersen RC, Xu YC, O'Brien PC, Waring SC, Tangalos EG, Smith GE, Ivnik RJ, Thibodeau SN, Kokmen E. Hippocampal atrophy and apolipoprotein E genotype are independently associated with Alzheimer's disease. Ann Neurol 1998;43(3):303-10.

[24] Jack CR, Jr., Slomkowski M, Gracon S, Hoover TM, Felmlee JP, Stewart K, Xu Y, Shiung M, O'Brien PC, Cha R, Knopman D, Petersen RC. MRI as a biomarker of disease progression in a therapeutic trial of milameline for AD. Neurology 2003;60(2):253-60.

[25] Jack CRJ, Petersen RC, Xu YC, Waring SC, P.C. OB, Tangalos EG, Smith GE, Ivnik RJ, Kokmen E. Medial temporal atrophy on MRI in normal aging and very mild Alzheimer's disease. Neurology 1997;49:786-94. 
[26] Killiany RJ, Gomez-Isla T, Moss M, Kikinis R, Sandor T, Jolesz F, Tanzi R, Jones K, Hyman BT, Albert MS. Use of structural magnetic resonance imaging to predict who will get Alzheimer's disease. Ann Neurol 2000;47(4):430-9.

[27] Kohler S, Black SE, Sinden M, Szekely C, Kidron D, Parker JL, Foster JK, Moscovitch M, Winocour G, Szalai JP, Bronskill MJ. Memory impairments associated with hippocampal versus parahippocampal-gyrus atrophy: an MR volumetry study in Alzheimer's disease. Neuropsychologia 1998;36(9):901-14.

[28] Kramer JH, Schuff N, Reed BR, Mungas D, Du AT, Rosen HJ, Jagust WJ, Miller BL, Weiner MW, Chui HC. Hippocampal volume and retention in Alzheimer's disease. J Int Neuropsychol Soc 2004;10(4):639-43.

[29] Laird NM, Ware JH. Random-effects models for longitudinal data. Biometrics 1982;38(4):963-74.

[30] Lehtovirta M, Soininen H, Laakso MP, Partanen K, Helisalmi S, Mannermaa A, Ryynanen M, Kuikka J, Hartikainen P, Riekkinen PJ, Sr. SPECT and MRI analysis in Alzheimer's disease: relation to apolipoprotein E epsilon 4 allele. J Neurol Neurosurg Psychiatry 1996;60(6):644-9.

[31] Littell RC, Milliken GA, Stroup WW, Wolfinger RD. SAS System for Mixed Models. Cary, NC, USA: SAS Institute Inc.; 1996.

[32] Lopez OL, Kuller LH, Becker JT, Dulberg C, Sweet RA, Gach HM, Dekosky ST. Incidence of dementia in mild cognitive impairment in the cardiovascular health study cognition study. Arch Neurol 2007;64(3):416-20.

[33] Mann DMA. The neuropathology of the amygdala in ageing and in dementia. In: Aggleton J, editor. The amygdala: neurobiological aspects of emotion, memory, and mental dysfunction. New York: Wiley; 1992. p 575-93. 
[34] Markesbery WR, Schmitt FA, Kryscio RJ, Davis DG, Smith CD, Wekstein DR. Neuropathologic substrate of mild cognitive impairment. Arch Neurol 2006;63(1):3846.

[35] Mizuno K, Wakai M, Takeda A, Sobue G. Medial temporal atrophy and memory impairment in early stage of Alzheimer's disease: an MRI volumetric and memory assessment study. J Neurol Sci 2000;173(1):18-24.

[36] Moore PM, Baker GA. Validation of the Wechsler Memory Scale-Revised in a sample of people with intractable temporal lobe epilepsy. Epilepsia 1996;37(12):1215-20.

[37] Mori E, Yoneda Y, Yamashita H, Hirono N, Ikeda M, Yamadori A. Medial temporal structures relate to memory impairment in Alzheimer's disease: an MRI volumetric study. J Neurol Neurosurg Psychiatry 1997;63(2):214-21.

[38] Morris JC. The Clinical Dementia Rating (CDR): current version and scoring rules. Neurology 1993;43(11):2412-4.

[39] Morris JC, Heyman A, Mohs RC, Hughes JP, van Belle G, Fillenbaum G, Mellits ED, Clark C. The Consortium to Establish a Registry for Alzheimer's Disease (CERAD). Part I. Clinical and neuropsychological assessment of Alzheimer's disease. Neurology 1989;39(9):1159-65.

[40] Pennanen C, Kivipelto M, Tuomainen S, Hartikainen P, Hanninen T, Laakso MP, Hallikainen M, Vanhanen M, Nissinen A, Helkala EL, Vainio P, Vanninen R, Partanen K, Soininen H. Hippocampus and entorhinal cortex in mild cognitive impairment and early AD. Neurobiol Aging 2004;25(3):303-10.

[41] Petersen RC, Doody R, Kurz A, Mohs RC, Morris JC, Rabins PV, Ritchie K, Rossor M, Thal L, Winblad B. Current concepts in mild cognitive impairment. Arch Neurol 2001;58(12):1985-92. 
[42] Petersen RC, Jack CR, Jr., Xu YC, Waring SC, O'Brien PC, Smith GE, Ivnik RJ, Tangalos EG, Boeve BF, Kokmen E. Memory and MRI-based hippocampal volumes in aging and AD. Neurology 2000;54(3):581-7.

[43] Price JL, Ko AI, Wade MJ, Tsou SK, McKeel DW, Morris JC. Neuron number in the entorhinal cortex and CA1 in preclinical Alzheimer disease. Arch Neurol 2001;58(9):1395-402.

[44] Pruessner JC, Collins DL, Pruessner M, Evans AC. Age and gender predict volume decline in the anterior and posterior hippocampus in early adulthood. J Neurosci $2001 ; 21(1): 194-200$.

[45] Pruessner JC, Li LM, Serles W, Pruessner M, Collins DL, Kabani N, Lupien S, Evans AC. Volumetry of hippocampus and amygdala with high-resolution MRI and threedimensional analysis software: minimizing the discrepancies between laboratories. Cerebral Cortex 2000;10(4):433-42.

[46] Rosen WG, Mohs RC, Davis KL. A new rating scale for Alzheimer's disease. Am J Psychiatry 1984;141:1356-64.

[47] Simic G, Kostovic I, Winblad B, Bogdanovic N. Volume and number of neurons of the human hippocampal formation in normal aging and Alzheimer's disease. J Comp Neurol 1997;379(4):482-94.

[48] Squire LR. Memory and the hippocampus: A synthesis from findings with rats, monkeys, and humans. Psychological Review 1992;99:195-231.

[49] Teipel SJ, Mitchell A, Möller H-J, Hampel H. Improving linear modeling of cognitive decline in patients with mild cognitive impairment: Comparison of two methods. J Neural Transm Suppl 2007;Suppl. 72:241-7.

[50] Teipel SJ, Pruessner JC, Faltraco F, Born C, Rocha-Unold M, Evans A, Moller HJ, Hampel H. Comprehensive dissection of the medial temporal lobe in AD: 
measurement of hippocampus, amygdala, entorhinal, perirhinal and parahippocampal cortices using MRI. J Neurol 2006;253(6):794-800.

[51] Wechsler D. A standardized memory scale for clinical use. J Psychology 1945;19:8795.

[52] West MJ, Coleman PD, Flood DG, Troncoso JC. Differences in the pattern of hippocampal neuronal loss in normal ageing and Alzheimer's disease. Lancet 1994;344(8925):769-72. 


\section{Figure legends:}

Figure 1: Sampling distribution of multicentre effect sizes of the group difference in left hippocampus volume between $\mathrm{AD}$ and MCI patients

Samples of $\mathrm{n}=10$ were randomly drawn from a total of $117 \mathrm{AD}$ and $166 \mathrm{MCI}$ patients each, where sampling without replacement was iterated 10,000 times. The vertical red line indicates the mean of the sampling distribution, the vertical green line the effect size of the pooled analysis. The vertical black lines indicate the lower and the upper limits of the $95 \%$ confidence interval of the sampling distribution. The red graph indicates the standard normal distribution with the same mean and standard deviation as the sampling distribution of the multicentre effect size. For purposes of comparison between multicentre and monocentre effect sizes, the effect size of each monocentre analysis (red stars) was projected onto the plot (note that height of the stars was chosen arbitrarily and does not indicate frequency).

Figure 2: Sampling distribution of multicentre effect sizes of the group difference in left amygdala volume between AD and MCI patients

For legend see legend to figure 1.

Figure 3: Sampling distribution of multicentre effect sizes of the group difference in right hippocampus volume between $\mathrm{AD}$ and MCI patients

For legend see legend to figure 1.

Figure 4: Sampling distribution of multicentre effect sizes of the group difference in right amygdala volume between $\mathrm{AD}$ and MCI patients

For legend see legend to figure 1. 


\begin{tabular}{|c|c|c|}
\hline & $\mathrm{AD}$ & MCI \\
\hline No. of patients (women) ${ }^{1}$ & $113(68)$ & $150(71)$ \\
\hline Age $(\mathrm{SD})$ in years ${ }^{2}$ & $71.9(7.7)$ & $67.7(7.8)$ \\
\hline $\operatorname{MMSE}(\mathrm{SD})^{3}$ & $23.5(4.1)$ & $27.1(1.9)$ \\
\hline Years of education (SD) ${ }^{4}$ & $12.0(3.5)$ & $11.8(2.7)$ \\
\hline No. of patients (women) with ApoE genotype ${ }^{5}$ & $88(51)$ & $122(56)$ \\
\hline Age (SD) in years ${ }^{6}$ & $71.7(7.3)$ & $67.6(7.4)$ \\
\hline $\operatorname{MMSE}(\mathrm{SD})^{7}$ & $23.9(3.3)$ & $27.1(1.9)$ \\
\hline Years of education $(\mathrm{SD})^{8}$ & $12.2(3.5)$ & $11.7(2.7)$ \\
\hline
\end{tabular}

${ }^{1}$ significantly different between groups, $\chi^{2}=4.3,1 \mathrm{df}, \mathrm{p}=0.039$.

${ }^{2}$ significantly different between groups, $\mathrm{T}=-4.35,261 \mathrm{df}, \mathrm{p}<0.001$.

${ }^{3}$ significantly different between groups, Mann-Whitney-U 3180.5, p $<0.001$.

${ }^{4}$ Not significantly different between groups, $\mathrm{T}=-0.49,261 \mathrm{df}, \mathrm{p}=0.63$.

${ }^{5}$ Not different between subgroup with APO-E genotype and remaining subjects:

AD: gender: $\chi^{2}=0.82,1 \mathrm{df}, \mathrm{p}=0.37$;CI : gender: $\chi^{2}=0.54,1 \mathrm{df}, \mathrm{p}=0.46$.

${ }^{6}$ not different between subgroup and remaining subjects:

AD: age: $\mathrm{T}=0.45,111 \mathrm{df}, \mathrm{p}=0.66$; MCI: age: $\mathrm{T}=0.39,148 \mathrm{df}, \mathrm{p}=0.70$.

${ }^{7}$ not different between subgroup and remaining subjects:

AD: Mann-Whitney U = 1014, p = 0.55; MCI Mann-Whitney U 1592.5, $\mathrm{p}=0.57$.

${ }^{8}$ not different between subgroup and remaining subjects:

$\mathrm{AD}: \mathrm{T}=-1.31,111 \mathrm{df}, \mathrm{p}=0.19 ; \mathrm{MCI}: \mathrm{T}=1.34,148 \mathrm{df}, \mathrm{p}=0.18$ 
Table 2: Analysis of variance on the effects of diagnosis, age and gender on volumetric measures controlling for centre and rater effects

\begin{tabular}{|c|c|c|c|c|c|c|c|c|}
\hline \multirow[t]{2}{*}{ Effect } & \multicolumn{2}{|c|}{ 1. hippocampus } & \multicolumn{2}{|c|}{ 1. amygdala } & \multicolumn{2}{|c|}{ r. hippocampus } & \multicolumn{2}{|c|}{ r. amygdala } \\
\hline & $F_{259}^{1}$ & $\mathrm{p}$ & $F_{259}^{1}$ & $\mathrm{p}$ & $F_{259}^{1}$ & $\mathrm{p}$ & $F_{259}^{1}$ & $\mathrm{P}$ \\
\hline Diagnosis & 27.62 & 0.0001 & 6.26 & 0.02 & 23.63 & 0.001 & 2.67 & n.s. \\
\hline Age & 20.26 & 0.0001 & 0.58 & n.s. & 9.58 & 0.003 & 0.01 & n.s. \\
\hline Gender & 1.66 & n.s. & 14.26 & 0.001 & 4.65 & 0.04 & 16.56 & 0.001 \\
\hline
\end{tabular}

F-tests (1 denominator and 259 nominator degrees of freedom) of fixed effects from a mixed effects model with age, gender and diagnosis as fixed effects and rater and centre and the two way interactions between rater and centre on the one hand and age, gender and diagnosis on the other hand as random effects.

1. - left

r. - right

n.s.- not significant 
Table 3: Analysis of variance on the effects of cognitive measures and ApoE genotype on volumetric measures controlling for age, diagnosis, gender, centre and rater effects

\begin{tabular}{|c|c|c|c|c|c|c|c|c|}
\hline \multirow[t]{2}{*}{ Effect } & \multicolumn{2}{|c|}{ 1. hippocampus } & \multicolumn{2}{|c|}{ 1. amygdala } & \multicolumn{2}{|c|}{ r. hippocampus } & \multicolumn{2}{|c|}{ r. amygdala } \\
\hline & $F_{257}^{1}$ & $\mathrm{p}$ & $F_{257}^{1}$ & $\mathrm{p}$ & $F_{257}^{1}$ & $\mathrm{p}$ & & $\mathrm{P}$ \\
\hline Word learning & 1.83 & n.s. & 3.68 & 0.06 & 1.96 & n.s. & 4.82 & 0.03 \\
\hline Free recall words & 17.45 & 0.0001 & 4.49 & 0.035 & 9.74 & 0.002 & 7.64 & 0.006 \\
\hline WMS-RLM-I & 11.67 & 0.0007 & 3.91 & 0.05 & 8.24 & 0.005 & 5.24 & 0.023 \\
\hline WMS-RLM-D & 21.53 & 0.0001 & 1.42 & n.s. & 9.42 & 0.0024 & 9.21 & 0.003 \\
\hline Drawing & 0.01 & n.s. & 0.09 & n.s. & 0.03 & n.s. & 0.36 & n.s. \\
\hline Free recall drawings & 10.39 & 0.002 & 2.41 & n.s. & 11.86 & 0.0007 & 5.70 & 0.02 \\
\hline MMSE & 7.79 & 0.006 & 13.94 & 0.0002 & 7.16 & 0.008 & 13.90 & 0.0002 \\
\hline ADAScog & 6.36 & 0.02 & 9.32 & 0.0025 . & 2.95 & n.s. & 14.06 & 0.0002 \\
\hline \multirow[t]{2}{*}{ CDR-SB } & 4.74 & 0.03 & 2.72 & n.s. & 4.06 & 0.05 & 5.52 & 0.02 \\
\hline & $F_{204}^{1}$ & $\mathrm{P}$ & $F_{204}^{1}$ & $\mathrm{p}$ & $F_{204}^{1}$ & $\mathrm{p}$ & $F_{204}^{1}$ & $\mathrm{p}$ \\
\hline Apo E4 & 0.05 & n.s. & 0.10 & n.s. & 0.21 & n.s. & 0.70 & n.s. \\
\hline
\end{tabular}

F-tests of fixed effects from mixed effects models with cognitive measures/ApoE4 genotype, age, gender, diagnosis and interaction of diagnosis and cognitive measures/ApoE4 genotype as fixed effects and rater and centre and the two way interactions between rater and centre on the one hand and cognitive measures/ApoE4 genotype on the other hand as random effects.

WMS_RLM-I - Wechlser Memory revised logical memory test-immediate recall

WMS_RLM-D - Wechlser Memory revised logical memory test-delayed recall

CDR-SB - clinical dementia rating, sum of boxes

MMSE - Mini Mental State Examination

ADAScog- Alzheimer Disease Assessment Scale cognitive battery 
1. - left

r. - right

n.s.-not significant 
Table 4: Hippocampus and amygdala volumes within centres

\begin{tabular}{|c|c|c|c|c|c|c|c|}
\hline \multirow[t]{2}{*}{ Centre } & \multirow[t]{2}{*}{ Region } & \multicolumn{2}{|c|}{$\mathrm{N}$} & \multicolumn{2}{|c|}{ Mean volume (SD) } & \multirow[t]{2}{*}{ Cohen's d } & \multirow[t]{2}{*}{$\mathrm{p}$ value } \\
\hline & & MCI & $\mathrm{AD}$ & MCI & $\mathrm{AD}$ & & \\
\hline$I^{\S}$ & 1. hippocampus & 14 & 2 & 2311.19 (306.99) & $1618.52(333.06)$ & -1.8187 & 0.039 \\
\hline II & 1. hippocampus & 17 & 21 & $2122.39(373.34)$ & $1719.27(367.11)$ & -0.9653 & 0.0014 \\
\hline III & 1. hippocampus & 21 & 8 & $2106.76(283.84)$ & $1679.83(277.54)$ & -1.2616 & 0.0005 \\
\hline IV & 1. hippocampus & 8 & 5 & $1969.82(216.96)$ & $1408.01(424.83)$ & -1.3684 & 0.0054 \\
\hline V & 1. hippocampus & 2 & 2 & $2124.2(179.67)$ & $1794.02(830.2)$ & -0.6275 & 1 \\
\hline VI & 1. hippocampus & 11 & 10 & $1946.86(328.07)$ & $1433.9(490.1)$ & -1.0677 & 0.0112 \\
\hline VII & 1. hippocampus & 6 & 5 & $1624.86(190.74)$ & $1456.65(147.05)$ & -0.9049 & 0.1441 \\
\hline VIII & 1. hippocampus & 12 & 6 & $1803.69(423.01)$ & $1286.5(360.67)$ & -1.1103 & 0.0492 \\
\hline IX & 1. hippocampus & 12 & 19 & $1829.23(516.64)$ & $1530.64(404.78)$ & -0.6395 & 0.2087 \\
\hline$X$ & 1. hippocampus & 22 & 6 & $1826.77(368.14)$ & $1641.98(607.22)$ & -0.436 & 0.3412 \\
\hline XI & 1. hippocampus & 20 & 20 & $1762.37(448.6)$ & $1389.65(335.63)$ & -0.8584 & 0.0027 \\
\hline $\mathrm{XII}{ }^{\S}$ & 1. hippocampus & 5 & 9 & $1789.38(399.23)$ & $1815.28(381.79)$ & 0.0729 & 0.739 \\
\hline $\mathrm{I}$ & 1. amygdala & 14 & 2 & $622.52(134.46)$ & $626.18(161.23)$ & 0.0277 & 1 \\
\hline II & 1. amygdala & 17 & 21 & $594.95(141.63)$ & $498.5(123.6)$ & -0.6943 & 0.0545 \\
\hline III & 1. amygdala & 21 & 8 & $586.04(130.89)$ & $511.75(147.07)$ & -0.542 & 0.1432 \\
\hline IV & 1. amygdala & 8 & 5 & $606.95(103.88)$ & $438.31(114.49)$ & -1.2585 & 0.0404 \\
\hline $\mathrm{V}^{\S}$ & 1. amygdala & 2 & 2 & $548.04(26)$ & $443.73(24.53)$ & -1.6385 & 0.1213 \\
\hline VI & 1. amygdala & 11 & 10 & $501.66(148.51)$ & $465.79(95.82)$ & -0.2882 & 0.5262 \\
\hline $\mathrm{VII}^{\S}$ & 1. amygdala & 6 & 5 & $470.01(126.84)$ & $490.87(36.15)$ & 0.2239 & 0.8551 \\
\hline VIII & 1. amygdala & 12 & 6 & $501.86(150.19)$ & $405.63(83.46)$ & -0.7014 & 0.1601 \\
\hline IX & 1. amygdala & 12 & 19 & $594.58(269.69)$ & 491.73 (128.97) & -0.5192 & 0.441 \\
\hline $\mathrm{X}$ & 1. amygdala & 22 & 6 & $585.56(144.23)$ & $508.34(196.22)$ & -0.4949 & 0.2397 \\
\hline
\end{tabular}




\begin{tabular}{|c|c|c|c|c|c|c|c|}
\hline \multirow[t]{2}{*}{ Centre } & \multirow[t]{2}{*}{ Region } & \multicolumn{2}{|c|}{$\mathrm{N}$} & \multicolumn{2}{|c|}{ Mean volume (SD) } & \multirow[t]{2}{*}{ Cohen's d } & \multirow[t]{2}{*}{$\mathrm{p}$ value } \\
\hline & & MCI & $\mathrm{AD}$ & MCI & $\mathrm{AD}$ & & \\
\hline XI & 1. amygdala & 20 & 20 & $584.98(155.01)$ & $445.73(119.05)$ & -0.9068 & 0.0063 \\
\hline $\mathrm{XII}^{\S}$ & 1. amygdala & 5 & 9 & $481.75(136.85)$ & $509.51(127.98)$ & 0.2192 & 0.641 \\
\hline I & r. hippocampus & 14 & 2 & $2462.79(318.88)$ & $1516.05(333.24)$ & -2.1164 & 0.0262 \\
\hline II & r. hippocampus & 17 & 21 & $2060.85(203.36)$ & $1710.66(374.56)$ & -0.9911 & 0.0014 \\
\hline III & r. hippocampus & 21 & 8 & $2093.96(306.84)$ & $1589.46(410.35)$ & -1.2534 & 0.0047 \\
\hline IV & r. hippocampus & 8 & 5 & $1907.4(233.19)$ & $1536.14(482.26)$ & -0.9764 & 0.1073 \\
\hline V & r. hippocampus & 2 & 2 & $1938.61(435.31)$ & $1765.4(802.96)$ & -0.3227 & 1 \\
\hline VI & r. hippocampus & 11 & 10 & $1972.76(273.3)$ & $1365.97(401.69)$ & -1.3357 & 0.0025 \\
\hline VII & r. hippocampus & 6 & 5 & $1738.93(275.28)$ & $1529.17(112.56)$ & -0.8948 & 0.2733 \\
\hline VIII & r. hippocampus & 12 & 6 & $1713.84(476.95)$ & $1255.81(316.03)$ & -0.9636 & 0.061 \\
\hline IX & r. hippocampus & 12 & 19 & $1868.8(514.83)$ & 1599.37 (491.99) & -0.5282 & 0.1679 \\
\hline $\mathrm{X}$ & r. hippocampus & 22 & 6 & $1816.36(355.97)$ & $1426.19(412.98)$ & -0.9856 & 0.05 \\
\hline XI & r. hippocampus & 20 & 20 & $1810.15(439.79)$ & $1369.78(369.11)$ & -0.9602 & 0.0019 \\
\hline XII & r. hippocampus & 5 & 9 & $1942.92(375.57)$ & $1631.88(190.70)$ & -1.0386 & 0.205 \\
\hline I & r. amygdala & 14 & 2 & $644.22(129.07)$ & $470.08(174.42)$ & -1.2312 & 0.0807 \\
\hline II & r. amygdala & 17 & 21 & $595.58(120.04)$ & $502.35(125.81)$ & -0.7152 & 0.0859 \\
\hline III & r. amygdala & 21 & 8 & $591.03(165.9)$ & $500.29(93.05)$ & -0.5916 & 0.1304 \\
\hline IV & r. amygdala & 8 & 5 & $590.26(151.53)$ & $535.99(140.54)$ & -0.3769 & 0.4642 \\
\hline V & r. amygdala & 2 & 2 & $525.23(92.65)$ & $445.17(157.93)$ & -0.6939 & 0.4386 \\
\hline VI & r. amygdala & 11 & 10 & 491.05 (143.77) & $468.96(107.82)$ & -0.1763 & 0.6727 \\
\hline VII & r. amygdala & 6 & 5 & $468.56(83.02)$ & $450.08(70.01)$ & -0.2492 & 1 \\
\hline VIII & r. amygdala & 12 & 6 & $503.9(137.58)$ & $441.07(92.45)$ & -0.5016 & 0.5121 \\
\hline
\end{tabular}




\begin{tabular}{lccccccc}
\hline Centre & Region & \multicolumn{2}{c}{$\mathrm{N}$} & \multicolumn{2}{c}{ Mean volume (SD) } & Cohen's d & p value \\
& & MCI & AD & MCI & AD & & \\
\hline IX & r. amygdala & 12 & 19 & $540.18(261.24)$ & $550.34(113.75)$ & 0.0561 & 0.3944 \\
X & r. amygdala & 22 & 6 & $565.49(112.21)$ & $482.93(217.42)$ & -0.5877 & 0.1978 \\
XI & r. amygdala & 20 & 20 & $579.99(134.86)$ & $450.56(131.47)$ & -0.8812 & 0.0063 \\
XII & r. amygdala & 5 & 9 & $520(88.85)$ & $490.5(92.39)$ & -0.3319 & 0.386 \\
\hline
\end{tabular}

The p-values derive from Mann-Whitney U tests.

1. - left; r. - right

$\S$ - monocentre effect size outside the $95 \%$ confidence interval of the multicentre sampling distribution 
Table 5: Pooled and multicentre effect sizes

\begin{tabular}{lcccc}
\hline & 1. hippocampus & 1. amygdala & r. hippocampus & r. amygdala \\
\cline { 2 - 5 } mean of sampling & & & & \\
distribution & -0.8668 & -0.6035 & -0.9378 & -0.5159 \\
Pooled effect size & -0.8729 & -0.6030 & -0.9449 & -0.5187 \\
Sample size & 58 & 120 & 52 & 158 \\
\hline
\end{tabular}

1. - left; r. - right

$\S$ - total sample size required to show a significant difference between AD and MCI patients with $\mathrm{p}<0.05$ and $90 \%$ power. 
Figure 1: Sampling distribution of multicentre effect sizes of the group difference in left hippocampus volume between AD and MCI patients

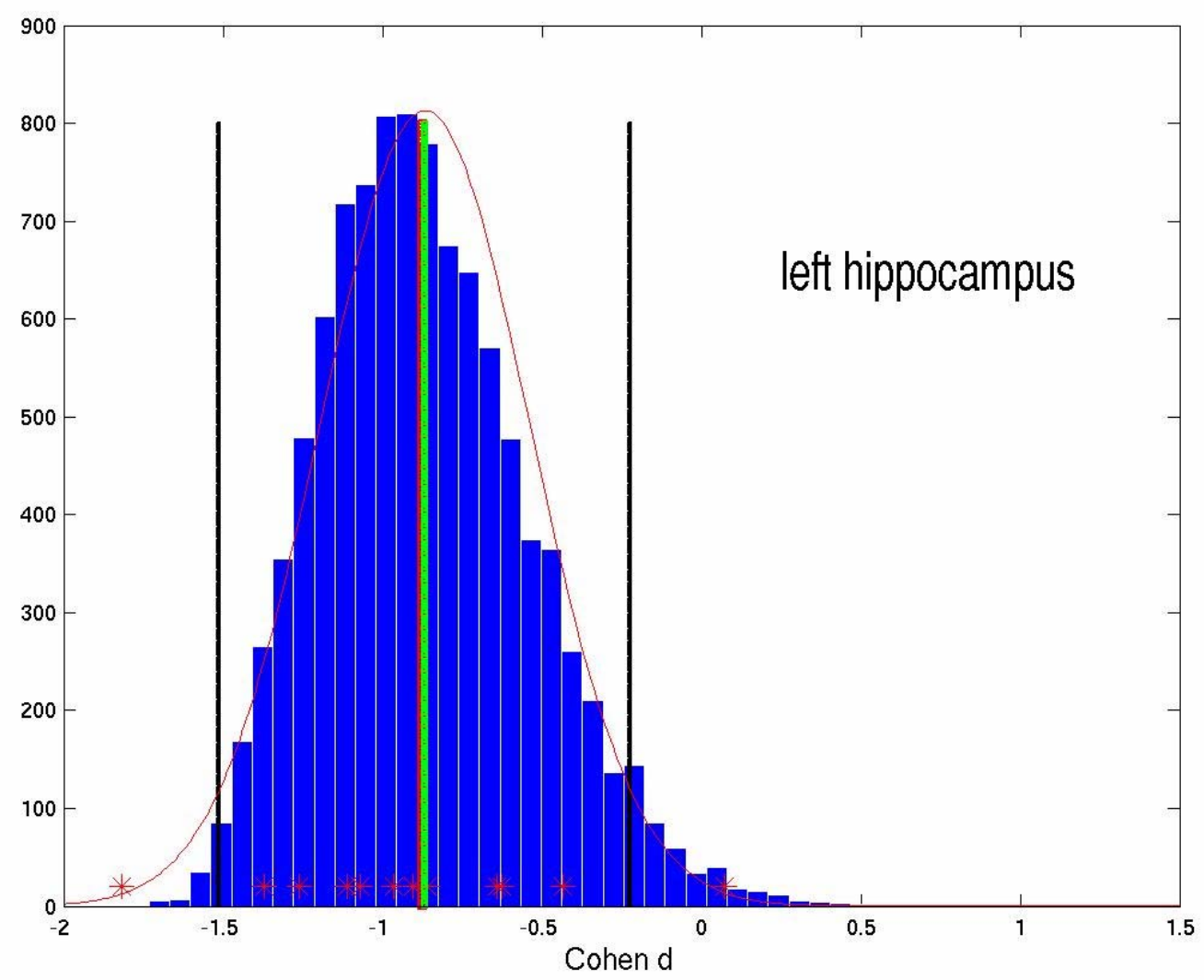


Figure 2: Sampling distribution of multicentre effect sizes of the group difference in left amygdala volume between $\mathrm{AD}$ and MCI patients

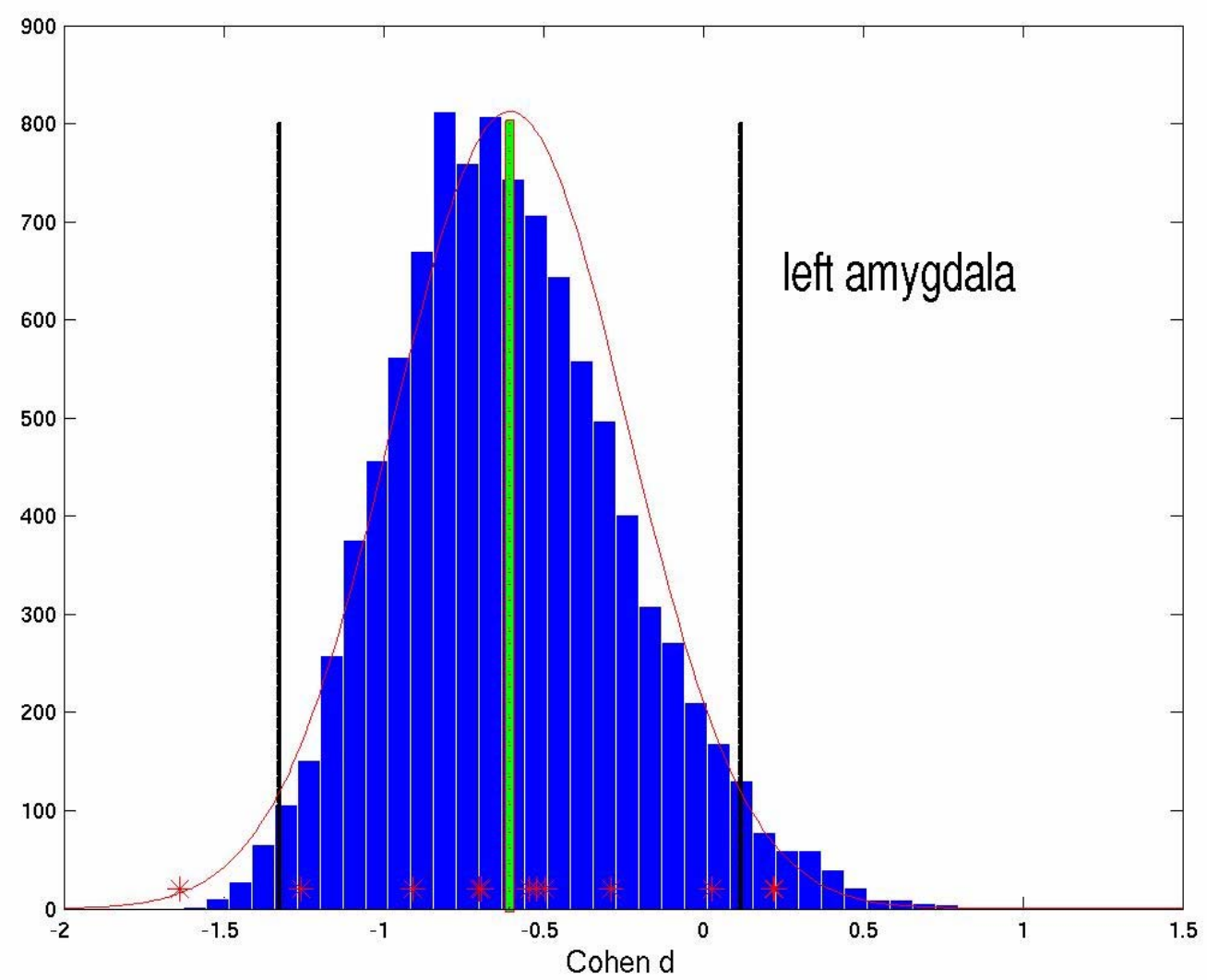


Figure 3: Sampling distribution of multicentre effect sizes of the group difference in right hippocampus volume between $\mathrm{AD}$ and MCI patients

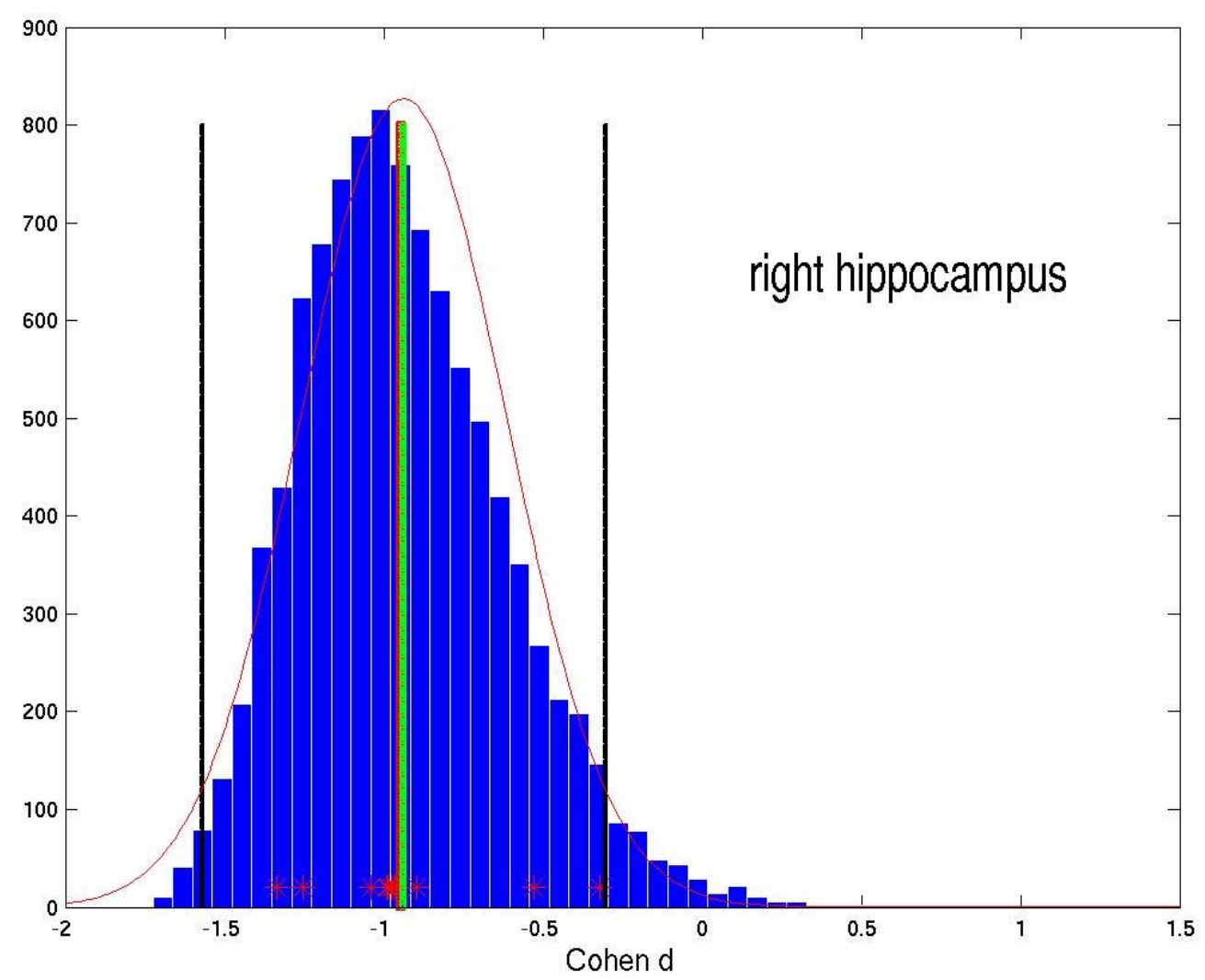


Figure 4: Sampling distribution of multicentre effect sizes of the group difference in right amygdala volume between $\mathrm{AD}$ and MCI patients

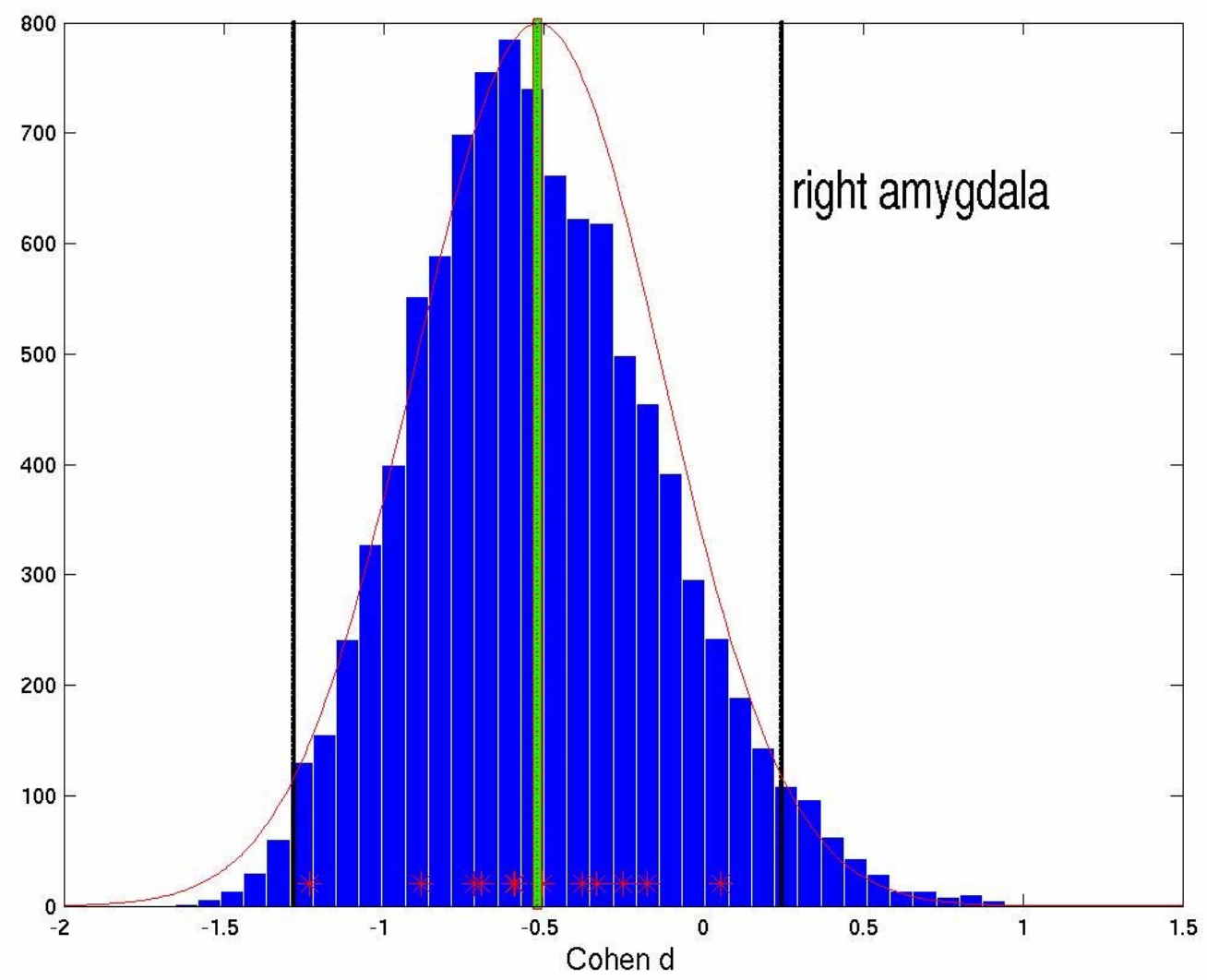

